\title{
Miniaturisation of combline filters for switched-mode power amplifier systems
}

\author{
E. Serebryakova, K. Blau, and M. Hein \\ Dept. for RF and Microwave Techniques, Technische Universität Ilmenau, Ilmenau, Germany
}

\begin{abstract}
This paper presents a coaxial combline filter for a class-S power amplifier for an operating frequency of $450 \mathrm{MHz}$. According to the measurement results, the filter provides a bandwidth of $26 \mathrm{MHz}$, an insertion loss of $0.55 \mathrm{~dB}$, and a second pass-band only at $2.8 \mathrm{GHz}$. As the filter dimensions are crucial for the envisaged application, different ways to miniaturise the filter are proposed and their features and disadvantages discussed.
\end{abstract}

\section{Introduction}

In wireless system architectures, the power amplifier (PA) is the final block in the transmitter chain. System efficiency is primarily determined by the characteristics of the PA. A switched-mode power amplifier can provide both high efficiency $(>70 \%)$ and high linearity. A class-S system, which permits very high efficient power amplification for arbitrary modulation schemes, consists of a one-bit modulator (e.g., a pulse length modulator), a switching amplifier and a bandpass reconstruction filter (Jayaraman et al. 1998). The output signal of the final stage of the class-S power amplifier includes noise-type signals from previous stages over a very broad frequency range. The band-pass filter has to provide not only very low insertion loss (an insertion loss of $1 \mathrm{~dB}$ reduces the overall efficiency by $20 \%$ ) and high frequency selectivity, but also a wide stop-band. The current-mode (pushpull) class-S configuration chosen for the final PA stage demands two filters for each signal way before signal combining and, therefore, the geometrical dimensions of these filters are crucial as well. The electrical as well geometrical constraints for the design of a filter for a class-S amplifier system can be satisfied advantageously by combline filters (CLF). This paper presents the coaxial realisation of a CLF, experimental results, and several ways to miniaturise it.

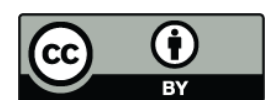

Correspondence to: E. Serebryakova (elena.serebryakova@tu-ilmenau.de)

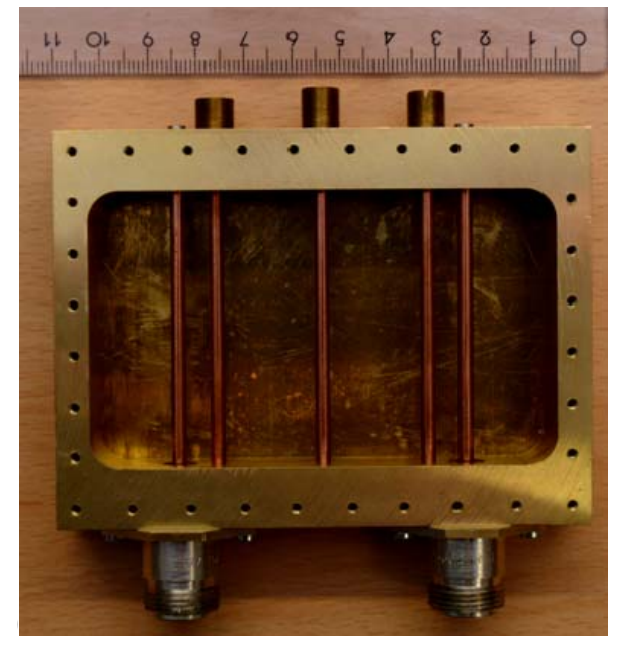

Fig. 1. Photograph of the fabricated combline filter.

\section{Combline filter}

The type of combline band-pass filters is well-known (Matthei et al., 1980). The resonators of such a filter consist of TEM-mode transmission line elements that are shortcircuited at one end and have a lumped capacitance between the other end and ground. With these lumped capacitors, the length of the transmission lines will be less than a quarter of the wavelength at resonance, and the resulting filter is geometrically compact. The second pass-band in a CLF occurs when the resonator line elements are somewhat over half a wavelength long. Therefore, the filter can be designed for very broad stop-band behaviour. Also, low losses can be achieved because the CLF can be fabricated without the use of dielectric support materials.

For the envisaged applications, the operating centre frequency of the filter was specified $f_{0}=450 \mathrm{MHz}$ and a bandwidth $\Delta f=10 \mathrm{MHz}$, the insertion loss less than $1 \mathrm{~dB}$, and the second pass-band higher than $2.5 \mathrm{GHz}$. According to these specifications, a third-order band-pass Butterworth filter without phase distortion in the pass-band was calcu-

Published by Copernicus Publications on behalf of the URSI Landesausschuss in der Bundesrepublik Deutschland e.V. 


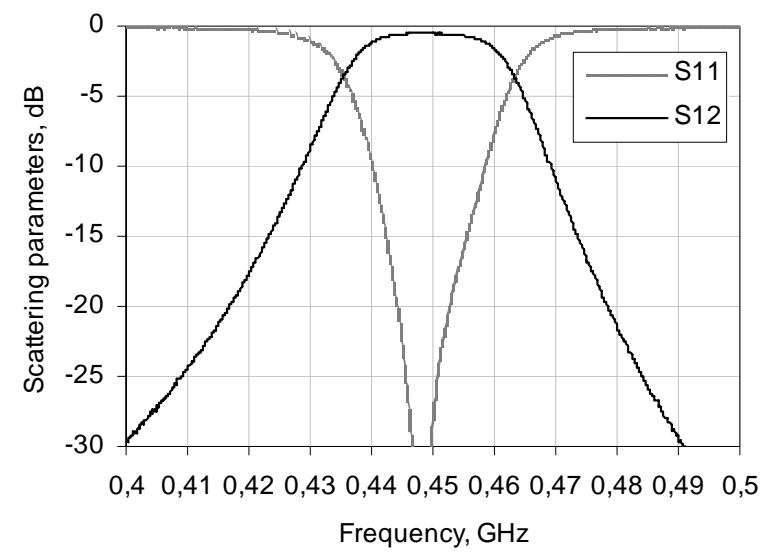

Fig. 2. The measured S-parameters: pass-band.

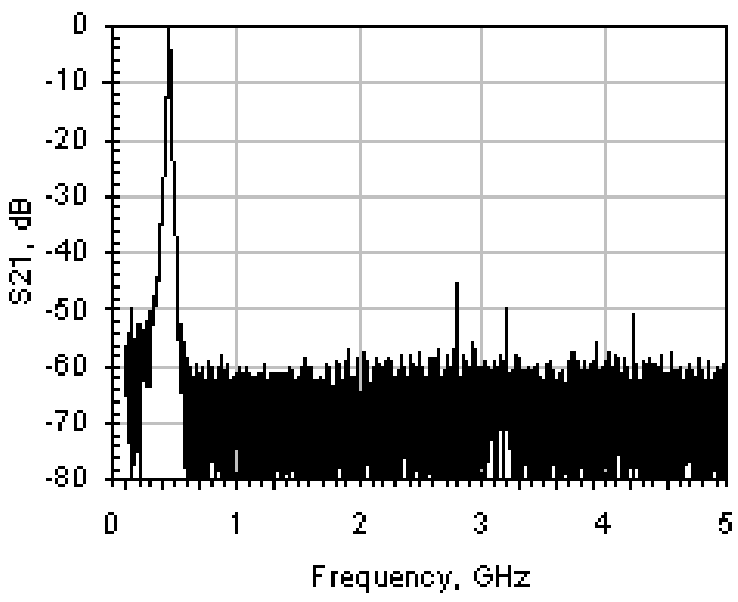

Fig. 3. The measured S-parameters: stop-band behavior.

lated and designed. To achieve the required stop-band behaviour, the electrical length $\theta_{0}$ of the resonators was chosen $30^{\circ}$. The CLF was realised as a coaxial structure without additional dielectric materials, in order to obtain quality factors of the coaxial resonators of higher than 2000 . The capacitances at the end of the resonators were implemented as distributed elements. Figures 1-3 show a photograph of the fabricated filter and the measured filter response. The size of the device is $105 \mathrm{~mm} \times 78 \mathrm{~mm} \times 30 \mathrm{~mm}$ or, in fractions of $\lambda$ : $0.16 \times 0.12 \times 0.05$. The filter provides a measured insertion loss of less than $0.55 \mathrm{~dB}$, a 3 -dB-bandwidth of $26 \mathrm{MHz}$, and a second pass-band at about $2.8 \mathrm{GHz}$.

\section{Miniaturisation of the combline filter}

Approaches to miniaturise a combline filter can be based on a reduction of the electrical length or the physical length. In the former case, the filter structure will be smaller in one dimension, and the stop-band will be broader. On the other hand,

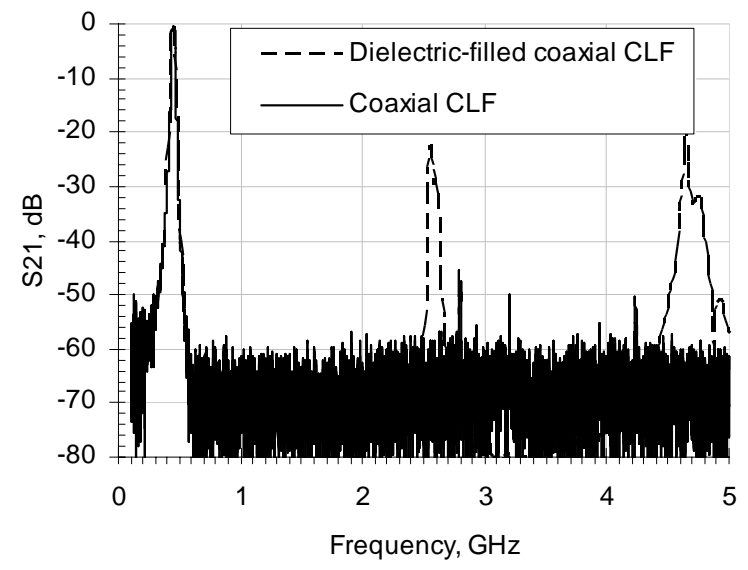

Fig. 4. Measurement results of the coaxial combline filter with PTFE (dotted curves) and without (solid curves).

shortening of the resonator line length degrades the unloaded quality factor and increases the pass-band insertion loss due to dissipation. Kurzrok gives an empirical relationship applicable to CLF resonators of constant cross section (Kurzrok, 1966):

$Q_{U L_{-} e f f} \cong \sin ^{2}\left(\theta_{0}\right) \cdot Q_{U L}$,

where $Q_{U L_{-} e f f}$ is the unloaded $Q$ of the shortened resonator, and $Q_{U L}$ is the unloaded $Q$ of the corresponding quarterwave resonator.

Consequently, the losses, which scale inversely with the quality factor of the resonators, increase according to the expression given by Cohn (1959):

$L_{0}=4.343 \cdot \frac{f_{0}}{\Delta f} \cdot \sum_{i=1}^{n} \frac{g_{i}}{Q_{U L_{-} e f f_{i}}},[d B]$

where $g_{i}$ are the normalised element values of the low-pass prototype. For our realisation, the shortened resonators with an electrical length of $22.9^{\circ}$ led to an increase of the insertion loss by fifty percent.

Another way to miniaturise a combline filter is to reduce the physical length of the resonators using dielectric-filled coaxial lines with fixed electrical length and cross-sectional dimensions. The added dielectric allows a reduction of the physical resonator length by the square root of its dielectric permittivity. Filters with shortened resonators and additional dielectric can be designed to have the same coupling between the resonators as the filter without dielectric. The dielectric material influences only the value of the capacitances at the end of the resonators and the insertion loss. A poly-tetrafluor-ethylene (PTFE)-filled CLF $(\varepsilon=2)$ was designed and fabricated as an example. The measurement results are shown in Fig. 4. The size of the device is $105 \mathrm{~mm} \times 62 \mathrm{~mm} \times 30 \mathrm{~mm}$. The filter provides the same low insertion loss and 3-dB-bandwidth as the original version, and a second pass-band at about $2.6 \mathrm{GHz}$. As a result, this 
method is very suitable for getting compact structures with low losses.

Yet another way to miniaturise the filter is a planar realisation. In this case, a wide variety of technologies exists, with the possibilities of using distributed or surface mounted capacitances. A planar CLF implementation with distributed capacitors at the end of the resonators is usually called "stepped impedance resonator" (SIR) (Ishizaki and Uwano, 1994). Such a realisation is, in general, very compact but the quality factors of the resonators are lower than in coaxial structures, usually $Q_{U L}<300$. Due to the inhomogeneous distribution of the electromagnetic fields in a planar structure (especially the current distribution), the attenuation due to dielectric losses and conductor losses is higher than in a coaxial transmission line, and radiation losses appear additionally. The wideband behaviour of a SIR depends on the impedance ratio (cross sectional dimensions of the transmission lines) and the length ratio (longitudinal dimensions).

\section{Conclusions}

A coaxial CLF for a class-S PA operating at $450 \mathrm{MHz}$ was designed, fabricated, and investigated experimentally. Several ways of miniaturisation were discussed in terms of a trade-off between size, insertion loss and stop-band behaviour; the particular choice depends on the specific filter requirements. If compactness is critical, planar structures or shortened resonators can be used. Otherwise, the dielectricfilled coaxial line implementation appears more suitable.
Acknowledgements. The authors would like to thank C. Hartmann and M. Huhn for valuable discussion and support. This work has been supported in part by the German Ministry of Education and Research within the class-S consortium of the focus programme "mobileGaN" (Grant 01BU0603).

\section{References}

Cohn, S. B.: Dissipation loss in Multiple-Coupled Resonators Filters, Proc. IRE, 47, 1342-1348, 1959.

Ishizaki, T. and Uwano, T.: A stepped impedance comb-line filter fabricated by using ceramic lamination technique, IEEE MTT-S 2, 617-620, 1994.

Jayaraman, A., Chen, P. F., Hanington, G., et al.: Linear HighEfficiency Microwave Power Amplifiers Using Band-pass DeltaSigma Modulators, IEEE Microwave and Guided-Wave Letters, 8, 3, 21-123, March 1998.

Kurzrok, R. M.: Design of Comb Line Band-Pass Filters, IEEE MTT, 14, 351-353, 1966.

Matthaei, G. L., Jones, E. L., and Young, L.: Microwave filters, impedance-matching networks, and coupling structures, Dedham, Mass, Artech House Books, 1980. 